\title{
Delta and theta activity during slow-wave sleep are associated with declarative but not with non-declarative learning in children with sleep-disordered breathing
}

\author{
Péter Simor ${ }^{1,2 *}$, Zsófia Zavecz ${ }^{3,4}$, Eszter Csábi $^{5}$, Pálma Benedek ${ }^{6}$, Karolina Janacsek ${ }^{4,7}$, Ferenc Gombos ${ }^{8}$ \\ and Dezsố Németh ${ }^{4,7}$ \\ ${ }^{I}$ Department of Cognitive Science, Budapest University of Technology and Economics, Budapest, Hungary \\ ${ }^{2}$ Nyiró Gyula Hospital, National Institute of Psychiatry and Addictions, Budapest, Hungary \\ ${ }^{3}$ Doctoral School of Psychology, Eötvös Loránd University, Budapest, Hungary \\ ${ }^{4}$ MTA-ELTE NAP B Brain, Memory and Language Research Group, Institute of Cognitive Neuroscience and \\ Psychology, Research Centre for Natural Sciences, Hungarian Academy of Sciences, Budapest, Hungary \\ ${ }^{5}$ Institute of Psychology, University of Szeged, Szeged, Hungary \\ ${ }^{6}$ Heim Pál Children's Hospital, Budapest, Hungary \\ ${ }^{7}$ Institute of Psychology, Eötvös Loránd University, Budapest, Hungary \\ ${ }^{8}$ Department of General Psychology, Pázmány Péter Catholic University, Budapest, Hungary
}

(Received: January 27, 2017; accepted: February 25, 2017)

\begin{abstract}
Sleep-disordered breathing (SDB) is a prevalent sleep disorder among young children and is associated with daytime impairments, such as behavioral dysregulation, affective symptoms, and reduced cognitive performance. Microstructural changes of non-rapid eye movement sleep, particularly the reduction of slow frequency oscillations during slow-wave sleep (SWS) might be associated with impaired learning among children with SDB. In this study, we investigated the associations between learning capacity, overnight memory retention, and post-learning, spectral power density of SWS within a clinical sample of children $(n=27)$ with SDB. Participants performed a declarative (the "War of the Ghosts") and a non-declarative (the "Alternating Serial Reaction Time") memory task at night, before their clinical (nighttime polysomnographic) evaluation. Memory retention was assessed in the morning. Overnight changes in performance in the declarative and non-declarative task were not related to relative spectral power measures of SWS. Nevertheless, declarative learning capacity was positively correlated with relative delta $(1.25-4 \mathrm{~Hz})$ and negatively with relative theta $(4.25-8 \mathrm{~Hz})$ power. Although statistical learning was not associated with spectral power, general skill learning was positively associated with delta and negatively associated with theta power. Associations in case of declarative learning remained significant beyond the influence of age; however, in case of general skill learning the associations with delta and theta power were explained by age. These findings indicate that among children with SDB, oscillations within the delta and theta band during SWS are associated with declarative learning capacity, but are independent from non-declarative, statistical learning.
\end{abstract}

Keywords: sleep-disordered breathing (SDB); declarative learning; implicit learning; statistical learning; EEG; oscillations

\section{HIGHLIGHTS}

- SWS delta and theta power are linked to declarative learning in children with SDB

- The associations between SWS power and learning are not explained by age

- SWS spectra are not associated with overnight changes in performance

- SWS spectra are not associated with non-declarative learning in children with SDB

\section{INTRODUCTION}

Sleep-disordered breathing (SDB) is a highly common complaint in prepubertal children with prevalence rates between 7\% and 12\% (Brunetti et al., 2001; Castronovo et al., 2003; Ersu et al., 2004; Ferreira et al., 2000), but in some cases reported up to $34.5 \%$ (Castronovo et al., 2003). SDB comprises a broad spectrum of breathing-related sleep problems from primary snoring to the most severe forms of obstructive sleep apnea (OSA) (Marcus, 2001). Whereas OSA, that is characterized by apnea, hypopnea, transient hypoxia, hypercarbia and related arousals during sleep is diagnosed in $1-3 \%$ of children (Ali, Pitson, and Stradling, 1993; Bixler et al., 2009), milder forms of SDB, in which sleep disruptions and impaired gas exchange are not detected, are largely underdiagnosed (Blunden, Lushington,

* Correspondence: Péter Simor, PhD, Department of Cognitive Science, Budapest University of Technology and Economics, Egry J. utca 1., T épület/504, 1111 Budapest, Hungary, E-mail: psimor@cogsci.bme.hu

This is an open-access article distributed under the terms of the Creative Commons Attribution License, which permits unrestricted use, distribution, and reproduction in any medium for non-commercial purposes, provided the original author and source are credited. 
Lorenzen, Martin, and Kennedy, 2005). Here we investigate how sleep disruptions affect cognitive functioning in SDB.

A growing number of studies indicate that moderate to severe OSA has a detrimental impact on children's behavior, affect, and cognitive performance (Beebe and Gozal, 2002; Blunden et al., 2005; Gottlieb et al., 2003; O'Brien et al., 2004). The latter is corroborated by findings linking symptoms of SDB to behavioral dysregulation (Rosen et al., 2004), inattention/hyperactivity (Chervin et al., 2002), as well as to impaired learning, attention, and executive function (Csábi, Benedek, Janacsek, Katona, and Nemeth, 2013; Gottlieb et al., 2004; Halbower et al., 2006; Kohler et al., 2009). These adverse effects might be driven by disrupted restorative functions of nighttime sleep and reduced oxygen delivery resulting in neuronal damage (Beebe and Gozal, 2002; Blunden and Beebe, 2006). Although a recent study (Hunter and Gozal, 2016) involving a large number of preschool-aged children showed that the clinical severity of SDB symptoms [e.g., apnea/hypopnea index (AHI), arousals, oxygen desaturation] is associated with poorer cognitive abilities in a dose-dependent manner (i.e., the more severe the symptoms are, the worse the performance is); converging evidence indicates that compared with healthy, non-snoring controls even milder forms of SDB, such as habitual snoring, are predictive of impaired cognitive and behavioral profile (Archbold, Giordani, Ruzicka, and Chervin, 2004; Bourke et al., 2011a, 2011b; Csabi et al., 2015). For instance, intellectual abilities and academic functions (Bourke et al., 2011a), declarative memory performance, and executive skills (Gottlieb et al., 2004), as well as parent-rated neurobehavioral functions (Bourke et al., 2011b) were similarly impaired in school-aged children with moderate-to-severe or mild SDB symptoms.

Nevertheless, it is not clear, whether in children with mild SDB, poorer behavioral and cognitive profiles are associated with abnormal nocturnal respiratory patterns, since studies have provided inconclusive results in this regard (Bourke et al., 2011a, 2011b; Hunter and Gozal, 2016). Although sleep fragmentation provoked by apneic events is considered to be another important mechanism that might lead to daytime cognitive impairments (Beebe and Gozal, 2002; Blunden and Beebe, 2006), data regarding the link between disrupted sleep and cognitive performance in mild SDB are scarce. Sleep macrostructure seems to be unaltered in children with SDB, but more subtle indices of homeostatic sleep regulation suggest that abnormal respiration might interfere with cortical, slow frequency oscillations during deep sleep, specifically during slow wave sleep (SWS) (Jussila et al., 2016; Kheirandish-Gozal et al., 2007), albeit findings are not absolutely conclusive (Yang et al., 2010). The lower rate of A1 subtype arousals as quantified by the cyclic alternating pattern (CAP) (Kheirandish-Gozal et al., 2007), and frontally reduced activity in slower frequencies $(<4 \mathrm{~Hz})$ during deep sleep (Jussila et al., 2016) suggest that specific neural oscillations are relatively attenuated in children with SDB.

The frontally localized A1 subtype of CAP (Ferri et al., 2008) such as low-frequency oscillations, indexed by delta (1-4 Hz) power (Cajochen, Foy, and Dijk, 1999; Munch et al., 2004), reflect the restorative capacity of the brain
(Mander et al., 2010), and seem to play an important role in memory consolidation (Ferri et al., 2008; Mander et al., 2013; Marshall, Helgadottir, Molle, and Born, 2006; Rasch and Born, 2013). In line with the role of SWS in memory consolidation (Guo, Igue, Malhotra, Stickgold, and Djonlagic, 2013) that adults suffering from OSA are characterized by diminished SWS and reduced overnight improvement in a verbal-associates task, compared with a healthy control group. Interestingly, the OSA group showed reduced SWS during the experimental night only, when presleep learning occurred. According to the authors, diminished post-training increase in slow-wave activity might have contributed to impaired memory consolidation during sleep.

The expression of slow frequency oscillations [more frequently quantified by electroencephalogram (EEG) spectral power] during SWS is strongly dependent on the integrity of the prefrontal cortex (Mander et al., 2013), and seems to be critical for the efficiency of cognitive functions that rely mainly on prefrontal and related (e.g., hippocampus) brain regions (Ferrara and De Gennaro, 2011; Mander et al., 2010, 2013). As a matter of fact, SDB in children seems to impinge specifically on tasks that involve sustained attention, executive functions, or declarative learning (Archbold et al., 2004; Bourke et al., 2011a; Csábi et al., 2013; Gottlieb et al., 2003). On the other hand, in case of an implicit, nondeclarative learning task that does not require (or might even benefit from the reduction of) cognitive control functions (Nemeth, Janacsek, Polner, and Kovacs, 2013 ) children with SDB showed equivalent performance to controls (Csábi et al., 2013, 2015). More specifically, Csábi et al. $(2013,2015)$ reported impaired declarative learning, but intact non-declarative learning in children with SDB. Furthermore, the patient and the control group showed similar overnight memory retention in both tasks, indicating intact consolidation in children with SDB (Csabi et al., 2015). Nevertheless, in this study, the association between task performance and polysomnographic measures was not examined.

In light of previous studies that reported attenuated SWSspecific slow frequency oscillations in children with SDB (Jussila et al., 2016; Kheirandish-Gozal et al., 2007), our aim was to investigate the associations between SWS spectral power, learning performance, and overnight memory retention within a group of children with SDB. To further explore the specificity of sleep-related cognitive impairments in SDB, we applied a declarative, verbal memory task and an implicit, non-declarative statistical learning task. We hypothesize that SWS spectral power is associated with memory retention in SDB. To the best of our knowledge, this is the first study investigating the relationship between SWS, learning performance, and memory retention in children with SDB.

\section{METHODS}

\section{Participants}

Twenty-seven children participated in the experiment. Age, breathing events during sleep, body mass index, and sleep 
Table 1. Age, breathing events during sleep, body mass index (BMI), and sleep parameters of participants

\begin{tabular}{lc}
\hline Variable & Mean $(S D)$ \\
\hline Age (years) & $8.52(2.12)$ \\
Gender (male, \%) & 59.25 \\
BMI (kg/m²) & $18.28(4.72)$ \\
Sleep efficiency (\%) & $87.44(6.99)$ \\
Relative wake duration (\%) & $12.22(6.64)$ \\
Relative S1 duration (\%) & $2.96(2.34)$ \\
Relative S2 duration (\%) & $42.04(9.26)$ \\
Relative S3 duration (\%) & $33.41(9.86)$ \\
Relative REM duration (\%) & $21.74(5.52)$ \\
AHI & $3.48(15.24)$ \\
Maximum desaturation (\%) & $5.29(7.16)$ \\
Desaturation index & $6.33(21.04)$ \\
Snore index & $25.61(42.05)$ \\
\hline
\end{tabular}

Note. AHI: apnea/hypopnea index, measured as the number of events per hour; desaturation index: measured as the number of desaturations per hour; snore index: measured as snoring events per hour.

parameters are listed in Table 1. All participants were reported to snore by their parents and underwent an overnight polysomnography (PSG) for clinical evaluation at the Sleep Disorders Laboratory of Heim Pál Children's Hospital, Budapest, Hungary. All these patients met the International Classification of Sleep Disorders criteria (American Academy of Sleep Medicine, 2014) for primary snoring $(N=23)$ or OSA $(N=4)$. The diagnostic criteria for Primary Snoring are complaint of snoring made by an observer (e.g., the parent). Polysomnographic monitoring in case of this disease demonstrates inspiratory or expiratory sounds often occurring for prolonged episodes during the total sleep time (this can be measured by snoring index), but no associated abrupt arousals, arterial oxygen desaturation, or cardiac disturbances. The diagnostic criteria of OSA are frequent episodes of obstructed breathing occur during sleep, and complaint of excessive sleepiness or insomnia. Polysomnographic monitoring demonstrates obstructive apneas (this is measured by the AHI), frequent arousals from sleep and arterial oxygen desaturation associated with the apneic episodes.

The snore index of the snoring patients $(M=25.52$, $S D=44.16$, range: $0-155)$ significantly differed from zero $[t(22)=2.77, p=.01]$. The AHI of the participants who had been diagnosed with OSA $(M=23.05, S D=37.60$, range: $1-79)$ did not significantly differ from zero $[t(3)=$ $1.27, p=.31$, probably due to the low number of patients $(N=4)$. Given that the neurobehavioral deficits characterizing children with primary snoring seem to be similar to those found in children with OSA (Gozal and O'Brien, 2004), we did not intend to examine the OSA and snoring subgroups separately. Nevertheless, apart from the main analyses, we performed a separate analyses for the primary snoring subgroup only (these analyses are presented in the Supplementary Material). The data of one subject was removed from the analyses in relation to the declarative task, and of another subject from the analyses of the nondeclarative task, due to lack of motivation to perform the specific task. All SDB patients were untreated prior to and during the experimental night. Informed written parental consent and verbal assent of the children were provided. Participants did not receive any financial compensation for their participation. Ethics approval was obtained by the Ethics Committee at Heim Pál Children's Hospital, Budapest.

\section{Tasks}

Declarative memory task. Declarative memory performance was measured by the "War of the Ghosts" test (Bartlett, 1932; Bergman and Roediger, 1999). This is a story recall test, which is widely used to measure declarative, episodic memory (Andreano and Cahill, 2006; Bartlett, 1932; Bergman and Roediger, 1999; Schwabe et al., 2009). In this test, children are asked to listen and repeat a story which consists of 36 information chunks. Based on the standardized scoring, 1 point is given if an information chunk is correctly recalled, and 0.5 points are given if it is only partly correct (capturing the gist of the sentences) (Bartlett, 1932; Csábi et al., 2013; Gauld and Stephenson, 1967).

Non-declarative memory task. We used the Alternating Serial Reaction Time (ASRT) Task to assess nondeclarative learning performance. In this task, a stimulus (a dog's head) appears in one of the four empty circles displayed in the middle of the screen and participants have to press the corresponding button as quickly and accurately as possible (Nemeth et al., 2010). The computer used was equipped with a special keyboard with four marked keys (Z, C, B and $\mathrm{M}$ on a QWERTY keyboard), each corresponding to one of the horizontally aligned circles. The task consisted of two sessions, the first session (Learning Phase) consisted of 25 blocks, and the second session (Testing Phase) consisted of 5 blocks. Each block consisted 85 key presses - the first 5 stimuli were random for practice purposes, then an eight-element alternating sequence (e.g., 1r4r3r1r, where numbers represent the four places on the screen, and $r$ represents an event randomly selected from the four possible places) repeated 10 times. A different ASRT sequence was selected for each participant based on a permutation rule so that each of the six unique permutations of the four repeating events occurred. Consequently, six different sequences were used across participants. Similarly to earlier studies (Nemeth et al., 2010), stimuli were presented $120 \mathrm{~ms}$ after the previous response (responseto-stimulus interval). Each block required about 1.5-2 min and the entire Learning Phase took approximately 40-50 $\mathrm{min}$, and the Testing Phase took approximately 10-15 min. Between blocks, participants received feedback about their overall reaction time (RT) and accuracy (ACC) on the screen and then rested 10-20 s before starting a new block.

Due to the structure of the sequences in the ASRT task, some triplets or runs of three consecutive events occur more frequently (high-frequency triplets) than others (lowfrequency triplets). For example, in the above illustration, 1_4, 2_3, 3_1 and 4_2 (where “_" indicates the middle element of the triplet) would occur often because the third element (bold numbers) could be derived from the sequence or could also be a random element. In contrast, $1 \_3$ or $4 \_1$ would occur less frequently because in this case, the third element could only be random. Note that the final event of 
high-frequency triplets is more predictable from the initial event when compared with the low-frequency triplets [also known as non-adjacent second-order dependency (Remillard, 2008)]. Therefore, before analyzing the data we determined whether each item was the last element of a high-frequency or low-frequency triplet. Out of the 64 possible triplets, the 16 high-frequency triplets occurred $62.5 \%$ of the time and the 48 low-frequency triplets occurred $37.5 \%$ of the time. Note that the final event of high-frequency triplets is more predictable from the initial event compared with the low-frequency triplets.

Previous studies have shown that as people practice the ASRT task, they come to respond more quickly and more accurately to the high-frequency triplets than low-frequency triplets, revealing statistical learning (Howard et al., 2004; Howard and Howard, 1997b; Janacsek, Fiser, and Nemeth, 2012; Nemeth et al., 2010; Song, Howard, and Howard, 2007). In addition, general skill learning is revealed in the ASRT task by the overall speed-up due to practice, irrespective of the triplet types. Thus, the ASRT task enables to measure both statistical and general skill learning.

Finally, it is important to note that the task remained implicit for the participants throughout the experiment. According to previous experiments with the ASRT task, even after an extended practice of 10 days, participants are not able to recognize the hidden sequence (Howard et al., 2004).

\section{Procedure}

PSG recordings were performed in the Sleep Disorders Laboratory of Heim Pál Children's Hospital, Budapest, Hungary. All children accomplished first the declarative and then the non-declarative task in two separate sessions, prior to sleep and after sleep. The order of the tasks was fixed. Memory performance was assessed at 7-9 p.m. in the evening (Learning Phase), and $12 \mathrm{hr}$ later after nighttime sleep, at 7-9 a.m. in the morning (Testing Phase). This study was performed within the frames of the clinical evaluation, therefore children spent only one night in the laboratory, and no adaptation night was applied.

\section{$P S G$}

The PSG was performed with the Somnomedics Somnoscreen plus device and software (Randersacker, Germany). PSG was configured to record EEG leads C4, C3 referenced to the mathematically linked mastoids (A2, A1) as well as bipolar EOG, chin EMG, ECG, snoring (by nasal cannula), respiratory effort signals, $\mathrm{SpO}_{2}$, pulse rate, and body position. EEG electrodes $(\mathrm{C} 4, \mathrm{C} 3, \mathrm{~A} 2, \mathrm{~A} 1)$ were placed in accordance with the 10-20 electrode placement system (Jasper, 1958). Children were also fitted with two EOG electrodes (left and right EOG channels), monitoring vertical and horizontal eye movements; two EMG electrodes (bipolar channels) for the chin, bipolar ECG electrodes; in addition to internal body position sensors, a pulse oximeter, a nasal flow thermistor (for measuring snoring), and thoracic and abdominal respiration sensors. $\mathrm{Ag} / \mathrm{AgCl} \mathrm{EEG}$ cup electrodes were fixed with Ten20 EEG conductive paste (Weaver and Company, Aurora, CO, USA). Hardware filters
( $-6 \mathrm{~dB}$ filters) were set between $0.3 \mathrm{~Hz}$ (high-pass) and $100 \mathrm{~Hz}$ (low-pass), signals were collected and digitized with $256 \mathrm{~Hz} /$ channel sampling rate (synchronous) with 8 bits resolution. Electrode impedances were kept below $6 \mathrm{k} \Omega$.

\section{Spectral analyses}

Sleep stages and conventional parameters of sleep macrostructure were scored in accordance with standardized criteria (Silber et al., 2007) by two experienced sleep researchers. Spectral analyses were performed by a custom-made software tool for full night sleep EEG analysis (FerciosEEGPlus ${ }^{\circledR}$, Ferenc Gombos 2008-2016). Overlapping $(50 \%)$, artifact-free four-second-epochs of all EEG derivations were Hanning-tapered and Fourier transformed using the FFT (fast Fourier transformation) algorithm to calculate the average power spectral densities for whole night SWS [non-rapid eye movement (NREM) Stage 3 sleep] between 1 and $25 \mathrm{~Hz}$. Since the absolute power values may be biased due to age-dependent differences of the thickness and conductivity of the skull (Carrier, Land, Buysse, Kupfer, and Monk, 2001), we applied the relative spectral power values. Relative spectral power values were obtained for each frequency bin (width: $0.25 \mathrm{~Hz}$ ) by dividing the absolute power of the given frequency bin with the total spectral power (the sum of the absolute power of the whole range of analysis between 1 and $25 \mathrm{~Hz}$ ). The relative power values reflect the relative contribution of a given frequency range to the total spectrum. To reduce the number of parameters, we summed up frequency bins to generate five frequency band windows: delta $(1.25-4 \mathrm{~Hz})$, theta (4.25-8 Hz), alpha $(8.25-11 \mathrm{~Hz})$, sigma $(11.25-15 \mathrm{~Hz})$, and beta $(15.25-25 \mathrm{~Hz})$ frequency bands. We have extracted these measures from SWS, because slow frequency oscillations are predominant during the deepest stage of sleep. Moreover, due to technical artifacts occurring in some participants during the last third of the night (comprising mainly Stage 2 and REM sleep), we have decided to exclude the analyses of Stage 2 periods and focus exclusively on SWS.

\section{Statistical analysis}

Statistical analyses were carried out with the Statistical Package for the Social Sciences version 22.0 (SPSS, IBM) and MATLAB (version 7.10.0.499, R2010a, The MathWorks Inc., Natick, MA). In case of the declarative learning task, we used three measures: evening score, morning score, and memory consolidation. The latter was obtained by subtracting the evening score from the morning score (higher scores indicating reduced forgetting). In case of the non-declarative learning task, to facilitate data processing, the blocks of ASRT were organized into epochs of five blocks. The first epoch contained blocks $1-5$, the second epoch contained blocks 6-10, etc. We calculated mean accuracy scores (ACCs) for all responses and median RTs for correct responses only; separately for high- and lowfrequency triplets and for each subject and each epoch. Note that for each response $(n)$, we defined whether it was a highor a low-frequency triplet by considering whether it was more or less predictable from the event $n-2$. For the 
analyses reported below, as in previous research (Howard and Howard, 1997a; Nemeth et al., 2010; Song et al., 2007), two kinds of low-frequency triplets were eliminated: repetitions (e.g., 222 and 333) and trills (e.g., 212 and 343). Repetitions and trills were low frequency for all participants and people often showed pre-existing response tendencies to them (Howard and Howard, 1997a; Howard et al., 2004). By eliminating them we attempted to ensure that any highvs. low-frequency differences are due to learning and not to pre-existing response tendencies.

For each epoch, a learning score was also calculated as the difference between triplet types in RT (RT for lowprobability triplets minus RT for high-probability triplets) and ACCs (ACC for high-probability triplets minus ACC for low-probability triplets). To evaluate performance changes due to statistical learning, we conducted repeated measures analyses of variance (ANOVAs - see detailed description below) separately for ACC and RT. Greenhouse-Geisser epsilon ( $\varepsilon$ ) correction was used if necessary. Original $d f$ values and corrected $p$ values (if applicable) are reported together with partial eta-squared $\left(\eta_{p}^{2}\right)$ as the measure of effect size. To investigate the offline (overnight) changes of statistical learning, we compared the ACCs and RTs from the last epoch of Session 1 (Epoch 5) and the epoch of Session 2 (Epoch 6 assessed in the morning). These variables were submitted to a repeated measures design ANOVA with TRIPLET (high- vs. low-frequency) and EPOCH (last epoch of Session 1 and the epoch of Session 2) as within-subject factors. Additionally, we subtracted the learning index of last epoch (fifth) of the evening session from the first epoch of the morning session (sixth epoch) (this way, the positive value shows overnight learning, and the negative shows forgetting) indexing memory consolidation in terms of ACC and RT.

Normality of data distribution was verified based on the kurtosis and skewness of the data as well as the Kolmogorov-Smirnov test. To study the associations between learning performance, overnight change (memory consolidation), and SWS spectral power, Pearson's correlation analyses were conducted. Spearman's correlation coefficient was used when normality was violated. To control for the confounding factor of age (that might affect learning as well as SWS spectral power), we applied a hierarchical linear regression analysis including age as a predictor in our models.

\section{RESULTS}

\section{Behavioral data}

Declarative memory (story recall). First, we verified whether immediate recall (at the evening) significantly differed from morning recall. According to the paired samples $t$-test, a significant difference emerged reflecting forgetting from evening to morning (mean evening score $=6.68$, $S D=4.32$; mean morning score $=5.46, S D=4.36$; $t(25)=2.721, p=.011)$.

Non-declarative memory (ASRT). We conducted a repeated measures ANOVA on the 5 epochs of the first session with TRIPLET (high- vs. low-frequency) and EPOCH (1-5) as within-subject factors and ACCs as the dependent variable. The main effect of TRIPLET was significant $\left[F(1,24)=43.96, \eta_{p}^{2}=.65, p<.001\right]$, indicating statistical learning, that is, higher ACCs for the highfrequency triplets compared with the low-frequency ones $(90.10 \%$ vs. $87.40 \%$, respectively). The main effect of EPOCH was also significant $\left[F(4,96)=4.17, \eta_{p}^{2}=.15\right.$, $p=.004]$, indicating that ACC decreased across epochs (Fig. 1A). The TRIPLET $\times$ EPOCH interaction showed a trend $\left[F(4,96)=2.17, \eta_{p}^{2}=.08, p=.077\right]$ : the ACC for high-frequency triplets decreased less, than for low-frequency triplets.

Regarding RT, we conducted a similar repeated measures ANOVA on the 5 epochs of the first session with TRIPLET (high- vs. low-frequency) and EPOCH (1-5) as withinsubject factors and RTs as the dependent variable. The main effect of TRIPLET was significant $[F(1,24)=$ $\left.61.20, \eta_{p}^{2}=.72, p<.001\right]$, indicating statistical learning, that is, shorter RTs for high-frequency triplets compared with the low-frequency ones. The main effect of EPOCH was also significant $\left[F(1.98,46.78)=73.04, \eta_{p}^{2}=.75\right.$, $p<.001$ ], due to reduced RTs across epochs, that reflects general skill learning. The TRIPLET $\times \mathrm{EPOCH}$ interaction was not significant $\left[F(2.67,63.53)=1.93, \eta_{p}^{2}=.07\right.$,
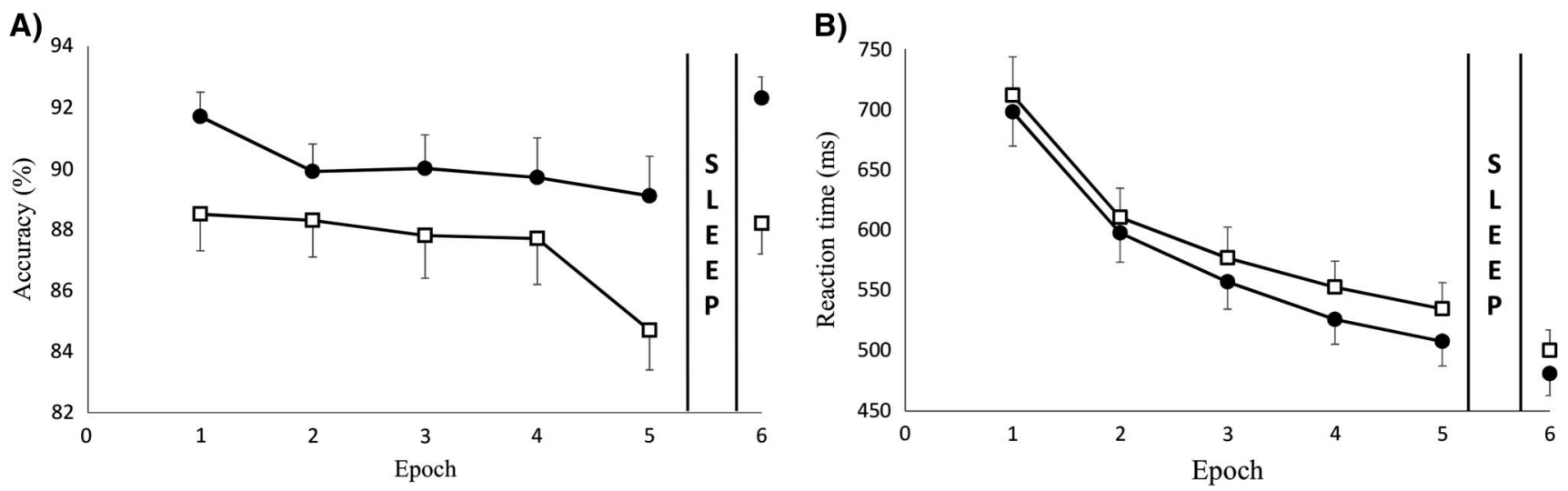

Fig. 1. The results of statistical learning on accuracy (A) and reaction time (B) measures. Accuracy (A) and RT for correct responses (B) can be seen as a function of epoch (1-6) and trial type (high- vs. low-frequency triplets). Black circles: high-frequency triplets. White squares: low-frequency triplets. The gap between the curves indicates the statistical learning performance. Error bars indicate standard error of the mean 
$p=.14]$, indicating that statistical learning was similar across the epochs (Fig. 1B).

To investigate the offline changes of statistical learning, we compared the ACCs of the last epoch of Session 1 (Epoch 5) with the ACCs of the epoch of Session 2 (Epoch 6 ). These variables were submitted to a repeated measures ANOVA with TRIPLET (high- vs. low-frequency) and EPOCH (last epoch of Session 1 and epoch of Session 2) as within-subject factors. The ANOVA yielded a significant main effect of TRIPLET $\left[F(1,22)=56.28, \eta_{p}^{2}=.72\right.$, $p<.001]$, indicating that, overall, participants were more accurate on high-frequency triplets compared with the lowfrequency ones. The main effect of EPOCH was also significant $\left[F(1,22)=17.80, \eta_{p}^{2}=.45, p<.001\right]$, due to more accurate responses in the morning compared with the evening session. The TRIPLET $\times$ EPOCH interaction was not significant $\left[F(1,22)=.12, \eta_{p}^{2}=.005, p=.74\right]$, indicating that statistical learning measured by $\mathrm{ACC}$, remained unchanged from the evening to the morning (Fig. 1A).

Regarding overnight changes in RTs, we compared the RTs from the last epoch of Session 1 (Epoch 5) with the RTs of the epoch of Session 2 (Epoch 6) by a similar repeated measures ANOVA. A significant main effect of TRIPLET $\left[F(1,22)=55.08, \eta_{p}^{2}=.72, p<.001\right]$, was found, indicating statistical learning, that is, RTs were shorter for high-frequency triplets compared with the low-frequency ones. The main effect of EPOCH was also significant $\left[F(1,22)=17.18, \eta_{p}^{2}=.44, p<.001\right]$, such that RTs decreased across epochs. The TRIPLET $\times$ EPOCH interaction was not significant $\left[F(1,22)=1.72, \eta_{p}^{2}=.07\right.$, $p=.20]$ indicating that statistical learning as measured by RT, remained unchanged from the evening to the morning (Fig. 1B).

\section{Associations between behavioral performance and SWS spectral power}

Declarative memory (story recall). SWS spectral power in the delta range showed a positive correlation with the evening story recall score $(r=.59, p=.001$, Fig. $2 A)$, whereas a negative correlation was found with the theta band $(r=-.65, p<.001$, Fig. $2 B)$. All other frequency bands showed non-significant $(p s>.68)$ correlations with the evening score. Similar correlations were found between the morning story recall score and band-wise spectral power measures (delta: $r=.472, p=.02$, theta: $r=-.52$, $p=.006$ ), all other $p \mathrm{~s}>.38$. No significant correlations were found between spectral power measures (all $p s>.59$ ) and overnight memory consolidation (i.e., the change in performance from evening to morning).

To control for the confounding factor of age that might influence both memory performance and SWS, we conducted a regression analysis with evening (immediate) story recall performance as the dependent factor, and age and SWS delta spectral power as separately entered independent variables. In the first model, performance in story recall was significantly associated with age (Std. beta $=.57, p=.002)$. In the second model where both age and delta spectral power were entered, age (Std. beta $=.40, p=.018)$, and delta power $($ Std. beta $=.46$, $p=.009$ ) were both significant predictors of immediate story recall. We conducted the same regression analysis with evening story recall performance as dependent variable, and age and SWS theta power as separately entered independent variables. In the final model, age was not significantly associated with story recall performance (Std. beta $=.29, p=.11)$, but theta power remained a significant predictor (Std. beta $=.-53, p=.006$ ). Both delta and theta power increased the explained variance of evening recall beyond the explained variance of age. Model parameters are detailed in Table 2.

Bin-wise correlations between story recall performance and power spectrum. To explore in more detail the oscillatory activity associated with declarative learning, we performed a post-hoc, bin-wise analyses within the delta and theta range in relation to evening memory performance. As plotted in Fig. 3, the spectral power in $1.25-1.5 \mathrm{~Hz}$ frequencies was positive, whereas frequency bins between 4 and $7 \mathrm{~Hz}$ were negatively associated with evening recall.

Non-declarative memory (ASRT). SWS spectral power measures were not associated with the statistical learning score in the evening (based on the last, fifth epoch) (all $p \mathrm{~s}>.22$ ) or in the morning session (all $p \mathrm{~s}>.41$ ) in terms of ACC. Moreover, spectral power measures were not associated with overnight consolidation (all $p \mathrm{~s}>.25$ ) of statistical
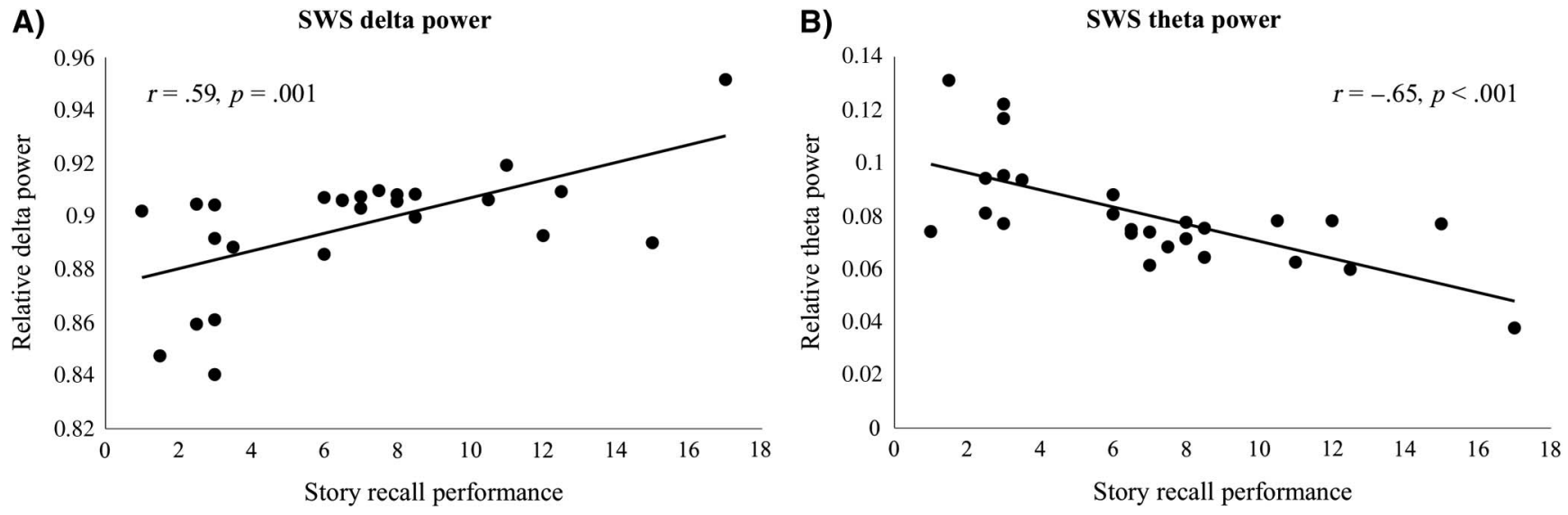

Fig. 2. Correlation between SWS delta (A) and theta (B) power spectrum and immediate (evening) story recall performance 
Table 2. Linear regression models with evening story recall performance as dependent variable

\begin{tabular}{lcccc}
\hline $\begin{array}{l}\text { Entered variables in } \\
\text { linear regression models }\end{array}$ & Std. beta & $t$ value & $p$ value & Model summary \\
\hline $\begin{array}{l}\text { Model } 1 \\
\text { Age }\end{array}$ & .57 & 3.41 & .002 & Adj. $R^{2}=.30, p=.002$ \\
Model 2 & .40 & 2.54 & .018 & \\
Age & .46 & 2.88 & .009 & Adj. $R^{2}=.46, p=.009$ \\
SWS delta power & .29 & 1.67 & .11 & \\
Model 3 & -.53 & -3.06 & .006 & Adj. $R^{2}=.48, p=.006$ \\
Age & & &
\end{tabular}

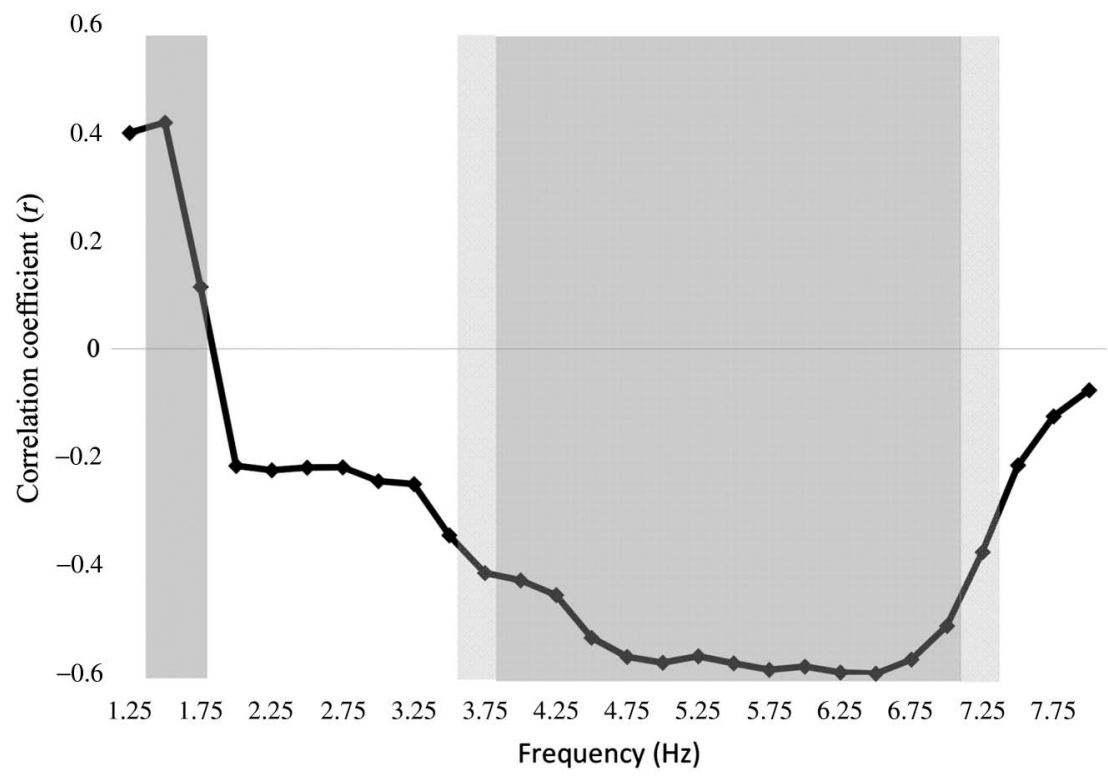

Fig. 3. Bin-wise correlation coefficients between 1 and $8 \mathrm{~Hz}$ spectral power and evening story recall performance. Gray background illustrates statistically significant $(p<.05)$ correlations and light gray background illustrates trend level $(p<.1)$

learning (overnight change in ACC). Similarly, no significant correlations emerged between statistical learning performance in the evening (all $p \mathrm{~s}>.25$ ) or in the morning session (all $p \mathrm{~s}>.11$ ) in terms of $\mathrm{RT}$, and spectral power measures were not associated with overnight consolidation (all $p \mathrm{~s}>.28$ ) (overnight change in RT).

Unlike statistical learning, SWS spectral power measures were associated with general skill learning in case of ACCs. Similarly to story recall, SWS spectral power in the delta range showed a positive correlation with the average ACCs (averaged across high- and low-frequency triplets) assessed in the evening (based on the last, fifth epoch, $r=.44$, $p=.028$ ), whereas a negative correlation was found with theta band power $(r=-.433, p=.03)$. All other frequency bands showed non-significant ( $p s>.45$ ) correlations with the average ACCs in the evening. Similarly, although stronger correlations were found between the morning ACCs and band-wise spectral power measures (delta: $r=.658$, $p=.001$; theta: $r=-.668, p<.001$, all other $p$ s $>.47$ ). No significant correlations were found between spectral power measures (all $p \mathrm{~s}>.25$ ) and overnight change in average ACCs (i.e., consolidation of general skill learning).
In case of general skill learning indexed by averaged RTs for high- and low-frequency triplets, no significant correlations emerged between skill learning and spectral power (all $p s>.10$ ). Neither we found significant correlations between the overnight RTs change and spectral power measures, although theta band power correlated with overnight change on a trend level $(r=-.391$, $p=.07$, all other $p$ s $>.12$ ).

Similarly to story recall, we controlled for the confounding factor of age that might influence both memory performance and SWS. First, we conducted a regression analysis with average evening ACCs as the dependent factor, and age and SWS delta spectral power as separately entered independent variables. In the first model, ACCs was significantly associated with age [Std. beta $=.51, p=.009 ;$ Adj. $\left.R^{2}=.23, F(1,23)=8.24 p=.009\right]$. In the second model, the influence of age remained significant [Std. beta $=.38$, $p=.05]$, but delta power was not a significant predictor [Std. beta $=.29, p=.14]$, of ACCs. This model was also significant [Adj. $R^{2}=.27, F(2,24)=5.51 p=.011$ ], but the $R^{2}$ change $(.07)$ was not significant $[F(1,22)=2.31$, $p=.14]$, indicating that the inclusion of delta power as a 
predictor did not significantly improve the model. We conducted the same regression analysis with average evening ACCs as dependent variable, and age and SWS theta power as separately entered independent variables. In the third model where both age and theta spectral power were entered, neither age (Std. beta $=.36, p=.11)$ nor theta power (Std. beta $=-.26, p=.24$ ) was significant predictors of ACCs. This model was also significant [Adj. $\left.R^{2}=.25, F(2,24)=4.93 p=.017\right]$, but the $R^{2}$ change (.05) was not significant $[F(1,22)=1.46, p=.24]$, indicating that the inclusion of theta power as a predictor did not significantly improve the model.

\section{Analysis of the primary snoring subjects}

To verify whether the above correlations were not produced due to impaired learning specifically within the OSA $(n=$ 4) subgroup, we performed the same analyses based on the data of the primary snoring subgroup only $(n=23)$. The exclusion of the OSA patients did not modify our results in case of the declarative and the non-declarative learning task. Delta power positively $(r=.62, p=.002)$ and theta power negatively $(r=-.67, p=.001)$ correlated with declarative learning capacity and general skill learning (delta range: $r=.59, p=.004$; theta range: $r=-.47, p=.03$ ). Whereas the associations in case of declarative learning were significant beyond the influence of age, the correlations between SWS spectral power and skill learning were not significant after controlling for age (see the Supplemental Material for a detailed description).

\section{DISCUSSION}

The principal aim of this study was to examine the associations between SWS-specific oscillatory activity and memory consolidation within a group of children with SDB. Inter-individual variability of post-learning, nighttime SWS spectral power did not predict overnight changes in performance either in case of a declarative or in a non-declarative learning task. Whereas no associations were found between SWS spectral power and indices of memory consolidation, delta and theta power were associated with declarative learning capacity. Delta power during post-learning SWS was positively associated with shortand long-term memory retention, assessed immediately after encoding, and after a nighttime sleep, respectively. On the other hand, faster oscillatory activity, indexed by the theta range was a negative correlate of short- and longterm memory performance. Given that both memory performance and SWS spectral power might be substantially influenced by cortical maturation, we also considered the effects of age. The associations between SWS power and declarative memory performance remained significant and considered for a large portion of the variance ( $16 \%$ for delta and $18 \%$ for theta) beyond the effects of age. In contrast, non-declarative statistical learning was not associated with SWS spectral power measures.

Our results indicate that slow frequency activity, in particular oscillations around $1 \mathrm{~Hz}$ are associated with better declarative learning capacity, whereas higher frequency activity between 4 and $7 \mathrm{~Hz}$ correlate with poorer performance among children with SDB. Two earlier studies (Jussila et al., 2016; Kheirandish-Gozal et al., 2007) reported attenuated slow frequency activity in children with SDB. Abnormal respiratory patterns could result in subtle changes in sleep physiology that might not be revealed by conventional macrostructural measures. Our findings suggest that the predominance of slow frequency $(\sim 1 \mathrm{~Hz})$ activity, as well as the reduction of faster $(4-7 \mathrm{~Hz})$ theta oscillations during SWS reflect better memory performance in children with SDB. Slow frequency activity of NREM sleep, quantified by the CAP A1 was consistently linked to better cognitive outcomes in healthy adults (Arico et al., 2010; Drago et al., 2011; Ferri et al., 2010) and children (Bruni et al., 2012). Given that slow frequency oscillations (with spectral power between 0.25 and $2.5 \mathrm{~Hz}$ ) are the main contributors of the visually detected CAP A1 subtypes (Ferri, Bruni, Miano, and Terzano, 2005), our findings indicating better declarative memory performance in relation to slower, and worse performance associated with faster frequencies, are in line with the concept of slow oscillations during SWS as sensitive biomarkers of healthy cognition (Tononi and Cirelli, 2006) or even neurodegeneration (Maestri et al., 2015). A large number of studies linked slow oscillations $(\sim 1 \mathrm{~Hz})$ to sleep-dependent memory consolidation (for an extensive review, see Rasch and Born, 2013); moreover, reduced increase in post-training SWS seems to be associated with impaired declarative memory consolidation in adults with OSA (Guo et al., 2013).

Nevertheless, in our sample SWS spectral power was not associated with overnight changes in performance, but only with general learning capacity. This finding might suggest that the associations between SWS spectral power and declarative learning are driven by traitdependent variance, instead of state-like effects of sleep on memory reprocessing. Such trait-dependent associations between cognitive measures and sleep-specific oscillations were not only reported for sleep spindles (Bodizs et al., 2005; Lustenberger, Maric, Durr, Achermann, and Huber, 2012; Ujma et al., 2014) but also for slow oscillations in case of parahippocampal-hippocampal recordings (Bódizs, Békésy, Szúcs, Barsi, and Halász, 2002). Although trait-dependent aspects might consider for our findings, associations between SWS power and memory performance could also be driven by learninginduced changes in EEG oscillations, as the expression of nocturnal slow frequency activity is particularly sensitive to previous learning experience (Molle, Marshall, Gais, and Born, 2004; Tononi and Cirelli, 2006). Therefore, state-like and trait-like effects in this study cannot be clearly discerned and should be explored in further investigations.

Whereas declarative learning was related to spectral power measures of SWS, non-declarative statistical learning, and overnight change in performance were not associated with SWS-specific oscillations. This finding coheres with earlier studies indicating that non-declarative statistical learning assessed by the ASRT does not benefit from sleep (Nemeth, Csábi, Janacsek, Varszegi, and Mari, 2012; Nemeth et al., 2010). More specifically, statistical learning 
did not produce off-line improvements in young and old participants and was not influenced by sleep (Nemeth et al., 2010). Furthermore, adults diagnosed with OSA (Csábi, Varszegi-Schulz, Janacsek, Malecek, and Nemeth, 2014; Nemeth et al., 2012) as well as children with SDB (Csábi et al., 2013) does not seem to exhibit impaired nondeclarative learning, suggesting that statistical learning captured by the ASRT is independent of the influence that sleep might have on cognitive functions. Although others reported sleep-dependent behavioral and neurophysiological effects (sleep-dependent memory consolidation) in case of similar probabilistic learning tasks (Durrant, Cairney, and Lewis, 2013; Durrant, Taylor, Cairney, and Lewis, 2011; Urbain et al., 2013), these tasks differ in their methodology and presumably, also in the underlying neural networks (Durrant et al., 2011, 2013; Janacsek, Ambrus, Paulus, Antal, and Nemeth, 2015; Nemeth, Janacsek, Király, et al., 2013; Urbain et al., 2013) that subtend them.

Moreover, statistical learning within the ASRT task is implicit and occurs without explicit awareness (Nemeth et al., 2010; Song et al., 2007). Several studies indicate that sleep-related benefits of memory consolidation are restricted to skill-learning paradigms that require attention, intentional learning (Wilhelm et al., 2011), explicit (verbally accessible) representations of the sequence structure (Robertson, Pascual-Leone, and Press, 2004; Song and Cohen, 2014), that are clearly not present in the ASRT task (Howard and Howard, 1997b).

General skill learning in terms of ACC, but not consolidation of skill learning was positively related to delta and negatively to theta power in SWS (see Supplementary Material), resembling the association found in case of declarative learning. This finding might be explained by at least partly overlapping cognitive processes underlying declarative learning and ACC performance measures. It has been previously shown that declarative learning is highly reliant on controlled, attention-dependent cognitive processes (Eichenbaum, 2000). Similarly, ACC performance measures have been suggested to rely on controlled, selective attentional processes to some extent (Prinzmetal, McCool, and Park, 2005). Nevertheless, the association between general skill learning and SWS spectra was explained by age, indicating that both ACC-related processes (Janacsek et al., 2012) and SWS activities (Buchmann et al., 2011) undergo robust age-related changes within this age range.

Some limitations of this study should be considered. Of all, although slow frequency oscillations were associated with declarative learning in our sample, we do not know if this correlation is specific to children with SDB, since we did not have a healthy control group. Given that we performed this study within the frames of a clinical evaluation, due to ethical and technical reasons, we did not include a baseline night without presleep learning experience. Although the associations between delta/theta power and learning capacity suggest a trait-like effect, trait-dependent and state-dependent effects cannot be differentiated since learning experience might also influence oscillatory activity of post-learning SWS. Our analyses focused on spectral power specifically during SWS, due to the predominance such oscillations during that sleep stage. Spectral activity during Stage 2 sleep might have also contributed to our analyses, however, due to a large number of technical artifacts in some participants during the last third of the night (comprising mainly Stage 2 and REM sleep), we have decided to focus exclusively on SWS sleep.

In spite of these limitations, this study indicates that among children with SDB, slow frequency oscillations within the delta and theta band during SWS are related to declarative learning capacity, but are independent of nondeclarative, statistical learning. These preliminary findings emphasize the relevance of oscillatory activity of SWS on specific cognitive processes and contribute to the characterization of cognitive functions and deficits of children with SDB. Future studies should further characterize which memory systems are specifically affected by fragmented sleep, and disentangle trait-dependent and state-dependent aspects of the interrelations between sleep and cognitive performance.

Authors' contribution: EC, DN, PB, and KJ designed the experiment, ZZ collected the data, PS, ZZ, and FG analyzed the data, PS, DN, ZZ, KJ, PB, EC, and FG wrote the manuscript. All the authors contributed equally to this work.

Conflict of interest: The authors declare no conflict of interest.

Ethics: This study was approved by the Ethics Committee at Heim Pál Children's Hospital, Budapest (Approval: Kut.32).

Acknowledgements: PS was supported by the European Union and the State of Hungary, co-financed by the European Social Fund in the framework of TAMOP 4.2.4 A/-111-2012-0001 "National Excellence Program" and by the János Bolyai Research Scholarship of the Hungarian Academy of Sciences. KJ was supported by the János Bolyai Research Scholarship of the Hungarian Academy of Sciences. The research was also supported by the Hungarian Brain Research Program (KTIA NAP 13-2-2015-0002).

\section{REFERENCES}

Ali NJ, Pitson DJ, and Stradling JR (1993). Snoring, sleep disturbance, and behaviour in 4-5 year olds. Arch Dis Child 68(3):360-366. doi:10.1136/adc.68.3.360

American Academy of Sleep Medicine (2014). International classification of sleep disorders. Darien, IL: American Academy of Sleep Medicine.

Andreano JM and Cahill L (2006). Glucocorticoid release and memory consolidation in men and women. Psychol Sci 17(6):466-470. doi:10.1111/j.1467-9280.2006.01729.x

Archbold KH, Giordani B, Ruzicka DL, and Chervin RD (2004). Cognitive executive dysfunction in children with mild sleep-disordered breathing. Biol Res Nurs 5(3):168-176. doi:10.1177/1099800403260261 
Arico D, Drago V, Foster PS, Heilman KM, Williamson J, and Ferri R (2010). Effects of NREM sleep instability on cognitive processing. Sleep Med 11(8):791-798. doi:10.1016/j.sleep. 2010.02.009

Bartlett FC (1932). Remembering: an experimental and social study. Cambridge, UK: Cambridge University.

Beebe DW and Gozal D (2002). Obstructive sleep apnea and the prefrontal cortex: towards a comprehensive model linking nocturnal upper airway obstruction to daytime cognitive and behavioral deficits. J Sleep Res 11(1):1-16. doi:10.1046/ j.1365-2869.2002.00289.x

Bergman ET and Roediger HL (1999). Can Bartlett's repeated reproduction experiments be replicated? Mem Cognit 27(6):937-947. doi:10.3758/BF03201224

Bixler EO, Vgontzas AN, Lin HM, Liao D, Calhoun S, Vela-Bueno A, Fedok F, Vlasic V, and Graff G (2009). Sleep disordered breathing in children in a general population sample: prevalence and risk factors. Sleep 32(6):731-736.

Blunden S, Lushington K, Lorenzen B, Martin J, and Kennedy D (2005). Neuropsychological and psychosocial function in children with a history of snoring or behavioral sleep problems. J Pediatr 146(6):780-786. doi:10.1016/j.jpeds.2005.01.043

Blunden SL and Beebe DW (2006). The contribution of intermittent hypoxia, sleep debt and sleep disruption to daytime performance deficits in children: consideration of respiratory and non-respiratory sleep disorders. Sleep Med Rev 10(2):109118. doi:10.1016/j.smrv.2005.11.003

Bódizs R, Békésy M, Szúcs A, Barsi P, and Halász P (2002). Sleepdependent hippocampal slow activity correlates with waking memory performance in humans. Neurobiol Learn Mem 78(2):441-457. doi:10.1006/nlme.2002.4078

Bodizs R, Kis T, Lazar AS, Havran L, Rigo P, Clemens Z, and Halasz P (2005). Prediction of general mental ability based on neural oscillation measures of sleep. J Sleep Res 14(3):285292. doi:10.1111/j.1365-2869.2005.00472.x

Bourke R, Anderson V, Yang JS, Jackman AR, Killedar A, Nixon GM, Davey MJ, Walker AM, Trinder J, and Horne RS (2011a). Cognitive and academic functions are impaired in children with all severities of sleep-disordered breathing. Sleep Med 12(5):489-496. doi:10.1016/j.sleep.2010.11.010

Bourke RS, Anderson V, Yang JS, Jackman AR, Killedar A, Nixon GM, Davey MJ, Walker AM, Trinder J, and Horne RS (2011b). Neurobehavioral function is impaired in children with all severities of sleep disordered breathing. Sleep Med 12(3):222-229. doi:10.1016/j.sleep.2010.08.011

Brunetti L, Rana S, Lospalluti ML, Pietrafesa A, Francavilla R, Fanelli M, and Armenio L (2001). Prevalence of obstructive sleep apnea syndrome in a cohort of 1,207 children of southern Italy. Chest 120(6):1930-1935. doi:10.1378/chest.120.6.1930

Bruni O, Kohler M, Novelli L, Kennedy D, Lushington K, Martin J, and Ferri R (2012). The role of NREM sleep instability in child cognitive performance. Sleep 35(5):649656. doi:10.5665/sleep. 1824

Buchmann A, Ringli M, Kurth S, Schaerer M, Geiger A, Jenni OG, and Huber R (2011). EEG sleep slow-wave activity as a mirror of cortical maturation. Cereb Cortex 21(3):607-615. doi:10.1093/cercor/bhq129

Cajochen C, Foy R, and Dijk DJ (1999). Frontal predominance of a relative increase in sleep delta and theta EEG activity after sleep loss in humans. Sleep Res Online 2(3):65-69.
Carrier J, Land S, Buysse DJ, Kupfer DJ, and Monk TH (2001). The effects of age and gender on sleep EEG power spectral density in the middle years of life (ages 20-60 years old). Psychophysiology 38(2):232-242. doi:10.1111/1469-8986.3820232

Castronovo V, Zucconi M, Nosetti L, Marazzini C, Hensley M, Veglia F, Nespoli L, and Ferini-Strambi L (2003). Prevalence of habitual snoring and sleep-disordered breathing in preschool-aged children in an Italian community. $J$ Pediatr 142(4):377-382. doi:10.1067/mpd.2003.118

Chervin RD, Archbold KH, Dillon JE, Panahi P, Pituch KJ, Dahl $\mathrm{RE}$, and Guilleminault C (2002). Inattention, hyperactivity, and symptoms of sleep-disordered breathing. Pediatrics 109(3):449-456. doi:10.1542/peds.109.3.449

Csábi E, Benedek P, Janacsek K, Katona G, and Nemeth D (2013). Sleep disorder in childhood impairs declarative but not nondeclarative forms of learning. J Clin Exp Neuropsychol 35(7):677-685. doi:10.1080/13803395.2013. 815693

Csábi E, Benedek P, Janacsek K, Zavecz Z, Katona G, and Nemeth D (2015). Declarative and non-declarative memory consolidation in children with sleep disorder. Front Hum Neurosci 9:709. doi:10.3389/fnhum.2015.00709

Csábi E, Varszegi-Schulz M, Janacsek K, Malecek N, and Nemeth D (2014). The consolidation of implicit sequence memory in obstructive sleep apnea. PLoS One 9(10):e109010. doi:10.1371/journal.pone.0109010

Drago V, Foster PS, Heilman KM, Arico D, Williamson J, Montagna P, and Ferri R (2011). Cyclic alternating pattern in sleep and its relationship to creativity. Sleep Med 12(4):361366. doi:10.1016/j.sleep.2010.11.009

Durrant SJ, Cairney SA, and Lewis PA (2013). Overnight consolidation AIDS the transfer of statistical knowledge from the medial temporal lobe to the striatum. Cereb Cortex 23(10):2467-2478. doi:10.1093/cercor/bhs244

Durrant SJ, Taylor C, Cairney S, and Lewis PA (2011). Sleepdependent consolidation of statistical learning. Neuropsychologia 49(5):1322-1331. doi:10.1016/j.neuropsychologia.2011.02.015

Eichenbaum H (2000). A cortical-hippocampal system for declarative memory. Nat Rev Neurosci 1(1):41-50. doi:10.1038/ 35036213

Ersu R, Arman AR, Save D, Karadag B, Karakoc F, Berkem M, and Dagli E (2004). Prevalence of snoring and symptoms of sleep-disordered breathing in primary school children in Istanbul. Chest 126(1):19-24. doi:10.1378/chest.126.1.19

Ferrara M and De Gennaro L (2011). Going local: insights from EEG and stereo-EEG studies of the human sleep-wake cycle. Curr Top Med Chem 11(19):2423-2437. doi:10.2174/ 156802611797470268

Ferreira AM, Clemente V, Gozal D, Gomes A, Pissarra C, Cesar H, Coelho I, Silva CF, and Azevedo MH (2000). Snoring in Portuguese primary school children. Pediatrics 106(5):E64. doi:10.1542/peds.106.5.e64

Ferri R, Bruni O, Miano S, and Terzano MG (2005). Topographic mapping of the spectral components of the cyclic alternating pattern (CAP). Sleep Med 6(1):29-36. doi:10.1016/j. sleep.2004.06.010

Ferri R, Drago V, Arico D, Bruni O, Remington RW, Stamatakis $\mathrm{K}$, and Punjabi NM (2010). The effects of experimental sleep fragmentation on cognitive processing. Sleep Med 11(4):378385. doi:10.1016/j.sleep.2010.01.006 
Ferri R, Huber R, Arico D, Drago V, Rundo F, Ghilardi MF, Massimini M, and Tononi G (2008). The slow-wave components of the cyclic alternating pattern (CAP) have a role in sleep-related learning processes. Neurosci Lett 432(3):228231. doi:10.1016/j.neulet.2007.12.025

Gauld A and Stephenson GM (1967). Some experiments relating to Bartlett's theory of remembering. Br J Psychol 58(1-2):39-49. doi:10.1111/j.2044-8295.1967.tb01054.x

Gottlieb DJ, Chase C, Vezina RM, Heeren TC, Corwin MJ, Auerbach SH, Weese-Mayer DE, and Lesko SM (2004). Sleep-disordered breathing symptoms are associated with poorer cognitive function in 5-year-old children. $J$ Pediatr 145(4):458-464. doi:10.1016/j.jpeds.2004.05.039

Gottlieb DJ, Vezina RM, Chase C, Lesko SM, Heeren TC, Weese-Mayer DE, Auerbach SH, and Corwin MJ (2003). Symptoms of sleep-disordered breathing in 5-year-old children are associated with sleepiness and problem behaviors. Pediatrics 112(4):870-877. doi:10.1542/peds.112.4.870

Gozal D and O'Brien LM (2004). Snoring and obstructive sleep apnoea in children: why should we treat? Paediatr Respir Rev 5(Suppl. A):S371-S376. doi:10.1016/S1526-0542(04) 90066-8

Guo M, Igue M, Malhotra A, Stickgold R, and Djonlagic I (2013). The effect of obstructive sleep apnea on declarative memory consolidation. Sleep Med 14:e33-e34. doi:10.1016/j. sleep.2013.11.041

Halbower AC, Degaonkar M, Barker PB, Earley CJ, Marcus CL, Smith PL, Prahme MC, and Mahone EM (2006). Childhood obstructive sleep apnea associates with neuropsychological deficits and neuronal brain injury. PLoS Med 3(8):e301. doi:10.1371/journal.pmed.0030301

Howard DV and Howard JH Jr (1997a). Adult age differences in effects of learning instructions on serial pattern learning. Paper presented at the American Psychological Society Meetings, Washington, DC.

Howard DV, Howard JH Jr, Japikse K, DiYanni C, Thompson A, and Somberg R (2004). Implicit sequence learning: effects of level of structure, adult age, and extended practice. Psychol Aging 19(1):79-92. doi:10.1037/0882-7974.19.1.79

Howard JH Jr and Howard DV (1997b). Age differences in implicit learning of higher-order dependencies in serial patterns. Psychol Aging 12(4):634-656. doi:10.1037/0882-7974.12.4.634

Hunter SJ and Gozal D (2016). Effect of sleep-disordered breathing severity on cognitive performance measures in a large community cohort of young school-aged children. Am J Respir Crit Care Med 194(6):739-747. doi:10.1164/rccm.2015102099OC

Janacsek K, Ambrus GG, Paulus W, Antal A, and Nemeth D (2015). Right hemisphere advantage in statistical learning: evidence from a probabilistic sequence learning task. Brain Stimul 8(2):277-282. doi:10.1016/j.brs.2014.11.008

Janacsek K, Fiser J, and Nemeth D (2012). The best time to acquire new skills: age-related differences in implicit sequence learning across the human lifespan. Devel Sci 15(4), 496-505. doi:10.1111/j.1467-7687.2012.01150.x

Jasper H (1958). Report of the committee on methods of clinical examination in electroencephalography. Electroencephalogr Clin Neurophysiol 10(2):370-375. doi:10.1016/0013-4694 (58)90053-1

Jussila M, Saarenpaa-Heikkila O, Huupponen E, Lapinlampi AM, Penttala J, and Himanen SL (2016). Sleep apnea reduces the amount of computational deep sleep in the right frontopolar area in school-aged children. Clin Neurophysiol 127(5):21672174. doi:10.1016/j.clinph.2016.02.016

Kheirandish-Gozal L, Miano S, Bruni O, Ferri R, Pagani J, Villa MP, and Gozal D (2007). Reduced NREM sleep instability in children with sleep disordered breathing. Sleep 30(4):450-457. doi:10.1093/sleep/30.4.450

Kohler MJ, Lushington K, van den Heuvel CJ, Martin J, Pamula Y, and Kennedy D (2009). Adenotonsillectomy and neurocognitive deficits in children with sleep disordered breathing. PLoS One 4(10):e7343. doi:10.1371/journal.pone.0007343

Lustenberger C, Maric A, Durr R, Achermann P, and Huber R (2012). Triangular relationship between sleep spindle activity, general cognitive ability and the efficiency of declarative learning. PLoS One 7(11):e49561. doi:10.1371/journal.pone.0049561

Maestri M, Carnicelli L, Tognoni G, Di Coscio E, Giorgi FS, Volpi L, Economou NT, Ktonas P, Ferri R, Bonuccelli U, and Bonanni E (2015). Non-rapid eye movement sleep instability in mild cognitive impairment: a pilot study. Sleep Med 16(9):1139-1145. doi:10.1016/j.sleep.2015.04.027

Mander BA, Rao V, Lu B, Saletin JM, Lindquist JR, Ancoli-Israel S, Jagust W, and Walker MP (2013). Prefrontal atrophy, disrupted NREM slow waves and impaired hippocampaldependent memory in aging. Nat Neurosci 16(3):357-364. doi:10.1038/nn.3324

Mander BA, Reid KJ, Baron KG, Tjoa T, Parrish TB, Paller KA, Gitelman DR, and Zee PC (2010). EEG measures index neural and cognitive recovery from sleep deprivation. $J$ Neurosci 30(7):2686-2693. doi:10.1523/jneurosci.4010-09.2010

Marcus CL (2001). Sleep-disordered breathing in children. Am J Respir Crit Care Med 164(1):16-30. doi:10.1164/ajrccm.164. 1.2008171

Marshall L, Helgadottir H, Molle M, and Born J (2006). Boosting slow oscillations during sleep potentiates memory. Nature 444(7119):610-613. doi:10.1038/nature05278

Molle M, Marshall L, Gais S, and Born J (2004). Learning increases human electroencephalographic coherence during subsequent slow sleep oscillations. Proc Natl Acad Sci U S A 101(38):13963-13968. doi:10.1073/pnas.0402820101

Munch M, Knoblauch V, Blatter K, Schroder C, Schnitzler C, Krauchi K, Wirz-Justice A, and Cajochen C (2004). The frontal predominance in human EEG delta activity after sleep loss decreases with age. Eur J Neurosci 20(5):1402-1410. doi:10.1111/j.1460-9568.2004.03580.x

Nemeth D, Csábi E, Janacsek K, Varszegi M, and Mari Z (2012). Intact implicit probabilistic sequence learning in obstructive sleep apnea. J Sleep Res 21(4):396-401. doi:10.1111/j.13652869.2011.00983.x

Nemeth D, Janacsek K, Király K, Londe Z, Németh K, Fazekas K, Adám I, Elemérné K, and Csányi A (2013). Probabilistic sequence learning in mild cognitive impairment. Front Hum Neurosci 7:318. doi:10.3389/fnhum.2013.00318

Nemeth D, Janacsek K, Londe Z, Ullman MT, Howard DV, and Howard JH Jr (2010). Sleep has no critical role in implicit motor sequence learning in young and old adults. Exp Brain Res 201(2):351-358. doi:10.1007/s00221-009-2024-x

Nemeth D, Janacsek K, Polner B, and Kovacs ZA (2013). Boosting human learning by hypnosis. Cereb Cortex 23(4):801-805. doi:10.1093/cercor/bhs068

O'Brien LM, Mervis CB, Holbrook CR, Bruner JL, Smith NH, McNally N, McClimment MC, and Gozal D (2004). 
Neurobehavioral correlates of sleep-disordered breathing in children. J Sleep Res 13(2):165-172. doi:10.1111/j.13652869.2004.00395.x

Prinzmetal W, McCool C, and Park S (2005). Attention: reaction time and accuracy reveal different mechanisms. J Exp Psychol Gen 134(1):73-92. doi:10.1037/0096-3445.134.1.73

Rasch B and Born J (2013). About sleep's role in memory. Physiol Rev 93(2):681-766. doi:10.1152/physrev.00032.2012

Remillard G (2008). Implicit learning of second-, third-, and fourth-order adjacent and nonadjacent sequential dependencies. Q J Exp Psychol (Hove) 61(3):400-424. doi:10.1080/ 17470210701210999

Robertson EM, Pascual-Leone A, and Press DZ (2004). Awareness modifies the skill-learning benefits of sleep. Curr Biol 14(3):208-212. doi:10.1016/j.cub.2004.01.027

Rosen CL, Storfer-Isser A, Taylor HG, Kirchner HL, Emancipator JL, and Redline S (2004). Increased behavioral morbidity in school-aged children with sleep-disordered breathing. Pediatrics 114(6):1640-1648. doi:10.1542/peds.2004-0103

Schwabe L, Römer S, Richter S, Dockendorf S, Bilak B, and Schächinger H (2009). Stress effects on declarative memory retrieval are blocked by a b-adrenoceptor antagonist in humans. Psychoneuroendocrinology 34(3):446-454. doi:10.1016/j. psyneuen.2008.10.009

Silber MH, Ancoli-Israel S, Bonnet MH, Chokroverty S, GriggDamberger MM, Hirshkowitz M, Kapen S, Keenan SA, Kryger MH, Penzel T, Pressman MR, and Iber C (2007). The visual scoring of sleep in adults. $J$ Clin Sleep Med 3(2):121-131.
Song S and Cohen LG (2014). Practice and sleep form different aspects of skill. Nat Commun 5:3407. doi:10.1038/ ncomms 4407

Song S, Howard JH Jr, and Howard DV (2007). Sleep does not benefit probabilistic motor sequence learning. J Neurosci 27(46):12475-12483. doi:10.1523/JNEUROSCI.2062-07.2007

Tononi G and Cirelli C (2006). Sleep function and synaptic homeostasis. Sleep Med Rev 10(1):49-62. doi:10.1016/j. smrv.2005.05.002

Ujma PP, Konrad BN, Genzel L, Bleifuss A, Simor P, Potari A, Körmendi J, Gombos F, Steiger A, Bódizs R, and Dresler M (2014). Sleep spindles and intelligence: evidence for a sexual dimorphism. J Neurosci 34(49):16358-16368. doi:10.1523/ jneurosci.1857-14.2014

Urbain C, Schmitz R, Schmidt C, Cleeremans A, Van Bogaert P, Maquet P, and Peigneux P (2013). Sleep-dependent neurophysiological processes in implicit sequence learning. $J$ Cogn Neurosci 25(11):2003-2014. doi:10.1162/jocn_a_00439

Wilhelm I, Diekelmann S, Molzow I, Ayoub A, Molle M, and Born J (2011). Sleep selectively enhances memory expected to be of future relevance. $J$ Neurosci 31(5):1563-1569. doi:10.1523/ jneurosci.3575-10.2011

Yang JS, Nicholas CL, Nixon GM, Davey MJ, Anderson V, Walker AM, Trinder JA, and Horne RS (2010). Determining sleep quality in children with sleep disordered breathing: EEG spectral analysis compared with conventional polysomnography. Sleep 33(9):1165-1172. doi:10.1093/sleep/ 33.9.1165 